\title{
Unraveling the mystery of spinosad resistance in insects
}

\author{
Jeffrey G. ScotT* \\ Department of Entomology, Comstock Hall, Cornell University, Ithaca, New York 14853 USA
}

(Received May 23, 2008; Accepted June 16, 2008)

\begin{abstract}
Spinosad is a new and highly promising insecticide, derived from the bacteria Saccharopolyspora spinosa, with efficacy against a wide range of insects. The mechanism of action of spinosad appears to be unique, with a primary site of attack being the nicotinic acetylcholine receptor (nAChR) and a secondary site of attack being $\gamma$ aminobutyric acid (GABA) receptors. Neural nAChRs are composed of five subunits, with a minimum of $2 \alpha$ s. Each subunit possesses a large N-terminal extracellular domain that includes the acetylcholine (ACh) binding site and four transmembrane domains (M1-4) with M2 contributing most of the amino acids that line the ion channel. Spinosad resistance has been selected for and characterized in several insect species. Generally, resistance is monofactorial, recessive and cannot be overcome by insecticide synergists. Spinosad resistance in the house fly maps to chromosome 1 and three nAChR subunit genes $(\alpha 5, \alpha 6$, and $\beta 3)$ are predicted to exist on chromosome 1 based on Drosophila/Musca homology maps. However, cloning and sequencing of Md $\alpha 5, M d \alpha 6$, and $M d \beta 3$ from susceptible and spinosad resistant strains of house fly found no differences that could be associated with resistance. Unraveling the mystery of spinosad resistance in house flies will require further study. (C) Pesticide Science Society of Japan
\end{abstract}

Keywords: spinosad resistance, nicotinic acetylcholine receptor, Musca domestica, Plutella xylostella, Drosophila melanogaster.

\section{Spinosad}

Spinosad (a mixture of spinosyns A and D, Fig. 1) is a new and highly promising insecticide, derived from the bacteria Saccharopolyspora spinosa, with efficacy against a wide range of insects. ${ }^{1-5)}$ The mechanism of action of spinosad appears to be unique, with a primary site of attack being the nicotinic acetylcholine receptor (nAChR) and a secondary site of attack being $\gamma$-aminobutyric acid (GABA) receptors. ${ }^{6-11)}$ Spinosad is thought to exert its toxicity primarily by activating a nAChR (nAChN subtype). ${ }^{9)}$ Studies showing that deletion of the $D \alpha 6 \mathrm{nAChR}$ results in strains of Drosophila melanogaster that are highly resistant to spinosad ${ }^{12)}$ confirms the importance of nAChRs in spinosad toxicity. This unique mechanism(s) of action suggests that resistance due to changes in the target sites of other major insecticides (i.e. acetylcholinesterase and voltage sensitive sodium channel) would not result in cross-resistance to spinosad. This appears true for house flies and the oriental fruit fly (Bactrocera dorsalis) where only modest levels of cross-resistance to spin-

\footnotetext{
* To whom correspondence should be addressed.

E-mail: jgs5@cornell.edu

(C) Pesticide Science Society of Japan
}

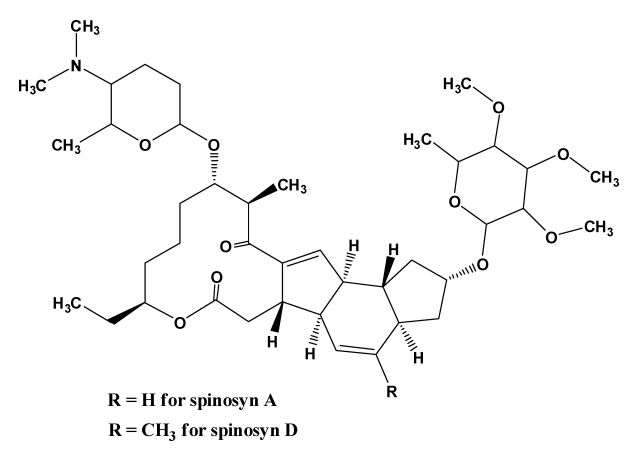

Fig. 1. Spinosad is a mixture of spinosyns A and D.

osad have been observed in laboratory strains and in field collected populations. ${ }^{13-17)}$ However, strains of Bactrocera dorsalis selected for resistance to organophosphates, carbamates or pyrethroids mechanisms unknown were up to 1000 -fold cross-resistant to spinosad. ${ }^{15)}$

\section{Nicotinic Acetylcholine Receptors}

Nicotinic acetylcholine receptors (nAChR) belong to the Cysloop superfamily of ligand-gated ion channels that include $\gamma$ aminobutyric acid (GABA)-gated channels, glycine receptors, glutamate-gated $\mathrm{Cl}^{-}$channels and 5-hydroxytryptamine type 
3 receptors. ${ }^{18)}$ nAChRs are composed of five transmembrane subunits arranged around a central water filled pore. A variety of subtypes of $\mathrm{nAChRs}$ arise from combinations of subunits that compose the channel-receptor complex. Although these subtypes display a range of different pharmacological and functional properties, they share some basic features. They have three main functional states: closed, open and closed desensitized. Exposure to the neurotransmitter ACh causes opening of the water-filled, cation-selective pore, followed (in about $2 \mathrm{msec}$ ) by closing to a nonconducting state. Prolonged exposure to low concentrations of nicotine produces desensitization, stabilizing the receptor in a closed state (unresponsive to agonists).

The diversity of nAChR subtypes contributes to the varied roles these receptors play in the central nervous system. By modulating activity-dependent events, nAChRs participate in fundamental aspects of synaptic plasticity that are involved in learning, memory and development. Thus, disruption of nAChRs has serious consequences, explaining why this target site has been exploited for the development of insecticides, including spinosad, and neonicotinoids (e.g. imidacloprid and thiamethoxam) ${ }^{19,20)}$ as well as the older insecticides nicotine, ${ }^{21)}$ cartap and bensultap. ${ }^{22)}$ Mutations or deletions of nAChRs can alter the toxicity of spinosad ${ }^{12)}$ or neonicotinoids. $^{23-25)}$ However, no mutation of a nAChR has yet been reported to alter the toxicity to both spinosad and neonicotinoids.

\section{Nicotinic Acetylcholine Receptor Subunits}

Neural nAChRs are composed of five subunits, with at least 2 $\alpha$ s. Receptors consisting of only $\alpha$ subunits are known in vertebrates, ${ }^{26)}$ but not in invertebrates. Each subunit possesses a large N-terminal extracellular domain that includes the ACh binding site and four transmembrane domains (M1-4) with M2 contributing most of the amino acids that line the ion channel. ${ }^{27)}$ The ACh binding site, in native and functional receptors, is located at the interface of two subunits, and is possibly contributed by three loops (loops A-C) of one subunit and by three (loops D-F) of the other. ${ }^{28)}$

The classification of nAChR subunits, as $\alpha$ or non- $\alpha$, is based on sequence similarity with nAChR subunits of the electric ray, Torpedo marmorata. A subunit possessing two adjacent cysteine residues, homologous to Cys192 and Cys 193 of $\alpha 1$ subunit of T. marmorata, which are known to be essential for ACh binding, is defined as a nAChR $\alpha$ subunit (YxCC motif). Subunits lacking this cysteine doublet are called non- $\alpha$ s, and include $\beta, \delta, \varepsilon$ or $\gamma^{29)}$ in vertebrates. In all animals neural nAChRs must have $\alpha$ and $\beta$ subunits. In vertebrates, muscle nAChRs have a subunit assembly of $(\alpha 1)_{2} \beta 1 \varepsilon \delta$ in the adult or $(\alpha 1)_{2} \beta 1 \gamma \delta$ in the fetus. ${ }^{30)}$ Insect nAChRs are only known to consist of combinations of $\alpha$ and $\beta$ subunits, ${ }^{31)}$ consistent with the fact that insects do not have cholinergic neuromuscular transmission. ${ }^{32)}$

\section{Genomics of nAChR Subunit Genes}

Within the insects, analysis of complete genome sequences revealed the presence of 10 ( $\alpha 1-7$ and $\beta 1-3), 10(\alpha 1-9$ and $\beta 1), 11$ ( $\alpha 1-9$ and $\beta 1-2)$ and 12 ( $\alpha 1-11$ and $\beta 1)$ nAChR subunits, in Drosophila melanogaster, Anopheles gambiae, Apis mellifera, and Tribolium castaneum, respectively. ${ }^{29,33-35)}$ These insect gene families are relatively small compared to those of vertebrates and nematodes, where 16 subunits have been identified in mammals and 17 in chicken. ${ }^{36)}$ In pufferfish, Fugu rubripes, 28 subunits $(16 \alpha$ and $12 \beta)$ were identified, making it the largest vertebrate $\mathrm{nAChR}$ gene family known to date. ${ }^{37)}$ In the Caenorhabditis elegans genome, 42 possible nAChR subunits were predicted from which 27 nAChR subunit transcripts have been determined. ${ }^{36)}$ The relatively small number of $\mathrm{nAChR}$ subunits in insects is compensated for by diversification due to alternative exon use and RNA editing ${ }^{29,38-40)}$ as described below.

Alternative splicing plays an important role in expanding protein diversity and is commonly employed to expand diversity of insect nAChRs. Alternative splicing plays an important role in $\mathrm{nAChR}$ diversification in $D$. melanogaster. For example, $D \alpha 4$ has an optional exon 2 and two alternative exon 4 s. There are also some very short transcripts observed $\left(\mathrm{D} \alpha 4^{\Delta \text { exon } 4}\right)$. The biological function of these shortened transcripts is not known, but they have been observed in both $D$. melanogaster $^{29)}$ and M. domestica. ${ }^{38,40)}$ The nAChR with the most alternative transcripts in D. melanogaster is $D \alpha 6$ (two alternative exon $3 \mathrm{~s}$ and three alternative exon $8 \mathrm{~s}$ ), where five different full length transcripts have been identified. ${ }^{41)}$ It appears use of alternative transcription may be conserved between species, as the same alternative exon use noted for $D \alpha 6$ was found in $M$. domestica, ${ }^{40)}$ and analysis of $\alpha 6$ nAChRs in An. gambiae, Bombyx mori, T. castaneum, Apis mellifera and 12 Drosophila species found all species had two exon 3s (except in A. mellifera) and three exons 8s (except in An. gambiae and B. mori $){ }^{42)}$ Although the effects of alternative splicing on $\mathrm{nAChR}$ function have yet to be fully determined, studies on other ion channels have demonstrated that splice variants encode functionally distinct proteins. ${ }^{43)}$ Since nAChR alternative exons contain binding loops B, D and E, as well as transmembrane region 2 , it seems very likely that these alternative transcripts produce proteins that are functionally distinct.

A-to-I RNA editing, catalyzed by adenosine deaminases acting on RNA (ADARs), is a pre-mRNA processing event that converts adenosine (A) to inosine (I) within short (approximately 9-15 bp), double stranded regions of RNA molecules. $^{44)}$ Because the inosine is recognized as guanosine $(\mathrm{G})$ by the cellular machinery, this editing functions as an A-to-G conversion. Therefore, A-to-I editing may result in amino acid substitutions, changes in protein functions or altering gene expression, by affecting RNA splicing, stability, and/or localization. ${ }^{29)}$ Interestingly, A-to-I editing seems to exclusively in- 


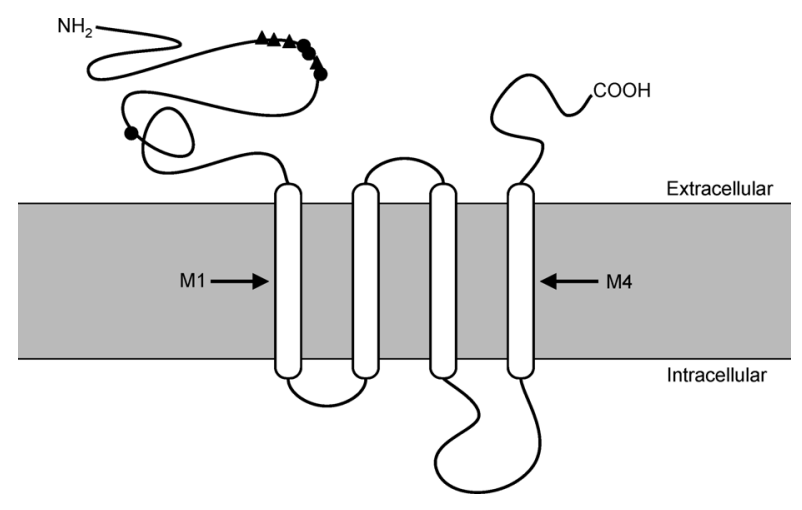

Fig. 2. Diagramatic representation of $M d \alpha 6$ showing approximate sites of RNA editing in exon 5 that result in an amino acid substitution. Sites that are edited in $<50 \%$, or $51-90 \%$ of the transcripts are indicated by a triangle and circle, respectively.

volve gene targets encoding rapid signaling components of the nervous system and frequently recodes amino acids critical for protein function. This may explain why ADAR deficient animals have unusual neurological phenotypes. ${ }^{29)}$ The relative frequency of A-to-I editing varies between sites for a given $\mathrm{nAChR}$. For example, A-to-I editing ranged from $3 \%$ to $100 \%$ at different sites within $M d \alpha 5^{38)}$

Grauso et al. first investigated A-to-I editing in the nAChR family and found seven edited sites in $D \alpha 6$ from $D$. melanogaster. ${ }^{41)}$ Hoopengardner et al. $^{45)}$ further identified seven, four and two A-to-I RNA editing sites in $D \alpha 5, D \beta 1$ and $D \beta 2$, respectively. We previously identified 12,13 , one and zero A-to-I RNA editing sites in house fly $M d \alpha 6, M d \alpha 5$, $M d \alpha 2, M d \beta 3$, respectively. ${ }^{38-40)}$ The sites of RNA editing for $\alpha 6$ subunit genes are conserved between $D$. melanogaster, $M$. domestica and $H$. virescens. ${ }^{34,40,41)}$ RNA editing is not randomly distributed across $\mathrm{nAChR}$ subunits, nor are the regions of the protein that have resulting amino acid substitutions the same between different subunits. For example, most RNA editing of $M d \alpha 6$ subunits occurs in the N-terminal extracellular

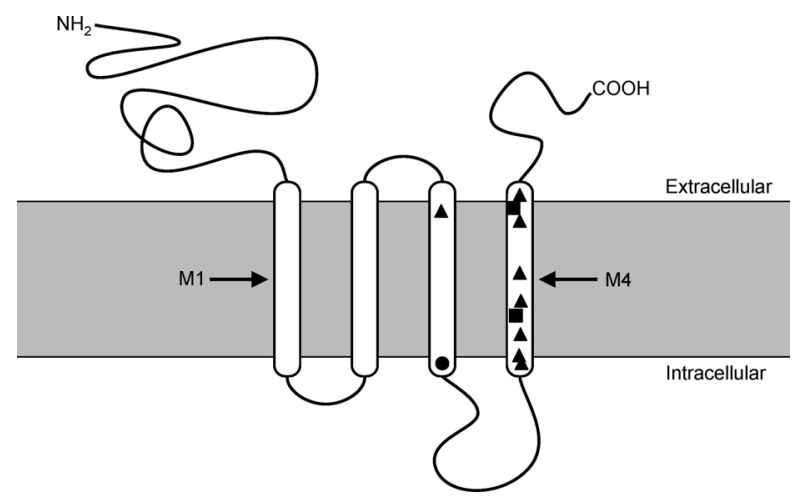

Fig. 3. Diagramatic representation of $\mathrm{Md} \alpha 5$ showing approximate sites of RNA editing that result in an amino acid substitution. Sites that are edited in $<50 \%, 51-90 \%$, or $100 \%$ of the transcripts are indicated by a triangle, circle, and square, respectively. loop (Fig. 2), while RNA editing of $M d \alpha 5$ occurs exclusively in transmembrane regions 3 and 4 (Fig. 3). RNA editing greatly diversifies the nAChR proteins found in insects. For example, over 30,000 D $\alpha 6$ proteins are theoretically possible through both alternative splicing and RNA editing. ${ }^{29)}$

\section{Characterization of Spinosad Resistance}

Spinosad resistance has been selected for and characterized in several insect species, although the molecular basis of the resistance is still not known. In Heliothis virescens a strain was selected that was 317-fold resistant when exposed to treated diet and 1070-fold resistant by topical application. ${ }^{46)}$ Resistance was incompletely recessive. ${ }^{47)}$ A strain of Plutella $x y$ lostella was collected in Hawaii and selected with spinosad which produced a strain that was 18,600 -fold resistant (leaf dip bioassay). Spinosad resistance could not be overcome by insecticide synergists, leading to the conclusion that metabolism mediated detoxification was probably not responsible for the resistance. ${ }^{48)}$ Spinosad resistance was controlled by one locus and was recessive in P. xylostella from Hawaii ${ }^{48)}$ and partially recessive in $P$. xylostella from Malaysia ${ }^{49)}$ and China. ${ }^{50)}$ Spinosad resistance has also been studied in Spodoptera exigua (85-fold), B. dorsalis (400-fold) and Musca domestica (below). Given the recessive nature of spinosad resistance, heterozygotes are very difficult to detect using insecticide bioassays. ${ }^{51)}$ If the mutation responsible for resistance could be identified, a powerful resistance monitoring technique could be developed that would differentiate homozygous susceptible, homozygous resistant and heterozygous individuals.

\section{Spinosad Resistance in the House Fly}

Selection of field collected house flies produced a highly spinosad resistant strain of house fly within just 10 generations of selection. The selections resulted in a strain (NYSPINR) $>150$-fold resistant to spinosad. Spinosad resistance in the NYSPINR strain was inherited as a recessive trait in agreement with results from other species (see above). The spinosad $\mathrm{LD}_{50}$ for the resistant strain was unchanged by pretreatment with the metabolism inhibitors PBO, DEF or DEM. Thus, it appears that the mechanism of resistance is not due to metabolic detoxification by monooxygenases, hydrolases or glutathione $S$-transferases, suggesting the resistance mechanism may be an altered target site. ${ }^{13)}$ Although spinosad appears to attack the $\mathrm{nAChR}$ and/or a GABA receptor, ${ }^{10,11)}$ and spinosad resistance in the house fly appears to be due to an altered target site, no cross-resistance has been detected to other insecticides, including those working at the nAChR (nicotine $^{13)}$ or imidacloprid (unpublished data)) or a GABA gated chloride channel (cyclodienes, abamectin or fipronil). ${ }^{13)}$ These studies support the hypothesis that spinosad has a unique mechanism of action. In order to produce a spinosad resistant strain that was genetically similar to the susceptible aabys strain, we crossed aabys with NYSPINR followed by a series 
of selections. ${ }^{13)}$ The resulting highly spinosad resistant strain was named rspin. ${ }^{13)}$

Linkage analysis revealed that spinosad resistance is on autosome $1 .^{13)}$ The only other known resistance gene on autosome 1 in house fly is CYP6D1-mediated (monooxygenase) resistance. Spinosad resistance does not appear to be caused by CYP6D1 because CYP6D1-mediated resistance can be greatly reduced by $\mathrm{PBO}$ and is inherited as a dominant trait, ${ }^{52)}$ neither of which is consistent with spinosad resistance in this strain.

Given the strong implications that $\mathrm{nAChRs}$ were the target site for spinosad, ${ }^{6,7,9,10,12)}$ and knowing that spinosad resistance was on autosome 1 in house fly, we hypothesized that mutations in one of the nAChR genes on house fly autosome 1 would have a mutation resulting in spinosad resistance. Using a Drosophila/Musca homology map ${ }^{53)}$ it was predicted that three house fly $\mathrm{nAChR}$ subunit genes $(\alpha 5, \alpha 6$ and $\beta 3)$ would be on house fly autosome 1 . We cloned and sequenced each of these cDNAs from susceptible and spinosad resistant house flies and our results are discussed below.

\section{Characterization of $M d \alpha 6$ from Susceptible and Spinosad Resistant House Flies}

The $M d \alpha 6$ cDNA was found to contain a 1470-bp ORF which encoded a 490 amino acid peptide that possesses a 22-residue long signal peptide. ${ }^{40)} M d \alpha 6$ has typical characteristics of nAChR $\alpha$ subunits, including a signal peptide, a long N-terminal extracellular domain, four hydrophobic transmembrane domains (TM1-4), and the YXCC motif in the N-terminal extracellular domain. The N-terminal domain possesses the Cys loop consisting of two cysteines separated by thirteen residues, a motif found in all ligand-gated ion channels ${ }^{27)}$ and the ACh binding site forming regions (loops A-F). ${ }^{28)} \mathrm{Md} \alpha 6$ has $97 \%, 89 \%, 84 \%$, and $73 \%$ identity with $\mathrm{D} \alpha 6$ of $D$. melanogaster, Agam $\alpha 6$ of An. gambiae, $\alpha 7-2$ of $H$. virescens and $\alpha$ 7-2 of $M$. persicae, respectively. ${ }^{40)}$

The genomic structure of $M d \alpha 6$ orthologs in $D$. melanogaster $(D \alpha \sigma)$ and An. gambiae (Agam $\alpha 6)$ have been determined, using the completed genome sequences. ${ }^{33,41)}$ Both contain 12 exons with alternative exons 3 ( $3 a$ and $3 b$ ) and 8 (8a, 8b and 8c in D. melanogaster, and 8b and 8c in An. gambiae). Five splice variants were identified in $D$. melanogaster. ${ }^{41)}$ Most of the splice variants of $M d \alpha 6$ were readily identified because they were nearly identical to $D \alpha 6$ transcripts. Based on the alternative use of exons 3 and 8 in $D \alpha 6$, and the classification criteria of Grauso et al., ${ }^{41)}$ six splicing variants were characterized from $M d \alpha 6$ : isoform I $(3 a+8 b)$, isoform II $(3 b+8 a)$, isoform III $(3 b+8 b)$, isoform IV $(3 a+3 b+8 a)$, isoform VI $(3 a+8 a)$, and isoform VII $(3 a+8 c){ }^{40)}$ Isoform II appeared to be the most common in house fly. The percentages of the isoforms were not significantly different between the head and thorax of individual flies from the same strain, or between heads or thoraces of susceptible (aabys) and spinosad resistant (rspin) house flies, except for isoforms II and III. ${ }^{40)}$ Isoform II was significantly lower and III was significantly higher in the thorax than in the head in rspin house flies $(P<0.05)$, but it was not significantly different from aabys house flies. Thus, alternative splicing of $M d \alpha 6$ does not appear to be involved in spinosad resistance. $^{40)}$

Unambiguous identification of A-to-I editing sites requires both genomic and cDNA sequences. Grauso et al. identified seven A-to-I RNA editing sites in $D \alpha 6$ (six in exon 5 and one in exon $6{ }^{41)}$ The editing sites in $M d \alpha 6$ exon 5 were investigated by examining the PCR amplified genomic sequence and 10 cDNA sequences (clones) from the same individuals $(n=3$ individuals and 30 clones). The nucleotide $\mathrm{A}$ was found in all of the $M d \alpha 6$ homologous positions in the genomic sequences, and $\mathrm{G}$ or $\mathrm{A}$ was found at eight sites in the cDNA sequences at a similar frequency to $D \alpha 6$. Sites 1 and 2 were considered to be adult-specific editing in $D \alpha 6{ }^{41)}$ Two new A-to-I editing sites in exon 5 of $M d \alpha 6$ were identified in this study and are located at nt 409 and 410 . They are edited with a frequency of $3-10 \%$ and $58-73 \%$, respectively. They resulted in amino acid substitutions from $\mathrm{N}$ to $\mathrm{D}$ or $\mathrm{G}$ or $\mathrm{S}$ in loop $\mathrm{E}$. A putative A-to-I editing site at nt 548 in $M d \alpha 6$ (=site 7 in $D$. melanogaster) was also examined by comparing only cDNA sequences. This site appears to be edited at a high frequency (65-79\%) and results in an $\mathrm{N}$ to $\mathrm{S}$ substitution in loop $\mathrm{F}$. In exon $8 \mathrm{~b}$, three additional $\mathrm{A} / \mathrm{G}$ polymorphic sites (putative Ato-I editing sites) were also identified at nt 851, 863 and 864, resulting in $\mathrm{E}$ to $\mathrm{G}$ and/or $\mathrm{Q}$ to $\mathrm{R}$ amino acid changes. These amino acid substitutions occurred in the N-terminus extracellular loop (near the important ligand binding domain) (Fig. 2). RNA editing was not significantly different either between head and thorax or between aabys and rspin strains $(P>0.05)$ and indicated that differences in A-to-I RNA editing of $M d \alpha 6$ between strains was not the cause of spinosad resistance in house flies. ${ }^{40)}$

Several polymorphisms were identified in $M d \alpha 6$ clones. ${ }^{40)}$ There were 17 and 12 synonymous single nucleotide polymorphisms (SNPs) in the ORF in the susceptible aabys and spinosad resistant rspin strains, respectively. There were an additional 13 and 21 nonsynonomous SNPs in the susceptible (aabys) and spinosad resistant (rspin) strains, respectively. Among the nonsynonomous SNPs, at least 8 were due to Ato-I RNA editing (discussed above). Both strains had transcripts in common, however, two polymorphic sites were unique to aabys and ten were unique to rspin. If changes in $M d \alpha 6$ were responsible for spinosad resistance, we would expect all transcripts in the rspin strain to be different from aabys. However, our results show no evidence of a selective sweep associated with the $M d \alpha 6$ locus in rspin (i.e. rspin contained transcripts also found in aabys). ${ }^{40)}$ Linkage analysis confirmed that $M d \alpha 6$ was on chromosome $1 .^{40)}$

Expression of $M d \alpha 6$ in the head, thorax and abdomen of adult flies was investigated using quantitative real-time PCR. There was no significant difference in the expression of $M d \alpha 6$ 
between spinosad susceptible and resistant strains, suggesting that differences in expression of $M d \alpha 6$ are not responsible for resistance. ${ }^{40)}$ These results contrast with the studies showing that deletion of $D \alpha 6$ results in a strain of Drosophila that is resistant to spinosad. ${ }^{12}$ ) Thus, although $D \alpha 6$ appears to be part of the spinosad target site, it is not altered in spinosad resistant house flies.

\section{Characterization of $M d \alpha 5$ from Susceptible and Spinosad Resistant House Flies}

The full length (4157-bp) cDNA of Md $\alpha 5$ from aabys flies consists of a 2343-bp ORF, a 1647-bp 5'UTR and a 167-bp $3^{\prime} \mathrm{UTR}^{38)}$ A 15 -nt deletion was found at a frequency of $14 \%$ (7/51 transcripts) in aabys flies and 21\% (3/14 transcripts) in rspin flies at nt 2041 to 2055, resulting in a loss of YLENL residues (681-685). Comparison with genomic DNA sequences revealed that this deletion was generated by an alternative use of donor (5') splice sites (gtattta or gtaatac) of intron 10. The use of the first donor splice site seems unique for $M d \alpha 5$ when compared to its orthologs in D. melanogaster, Anopheles gambiae and Aedes aegypti. ${ }^{33,41)}$ Comparisons of the different sequences in aabys and rspin demonstrate there is transcript variability within and between strains. However, given that rspin and aabys express many of the same transcripts, suggests that changes in the sequence of $M d \alpha 5$ are not responsible for spinosad resistance. ${ }^{38)}$

The 2343-bp ORF of $M d \alpha 5$ encodes a 781-amino acid proprotein that possesses a 27 -aa signal peptide. ${ }^{38)}$ Besides the signal peptide, the protein possesses typical characteristics of nAChR $\alpha$ subunits including a long N-terminal extracellular domain having the $\alpha$ subunit signature YXCC motif, the Cys loop domain and four hydrophobic transmembrane domains (TM1-4). The calculated molecular weight of Md $\alpha 5$ $(85.3 \mathrm{kDa})$ is greater than $\mathrm{D} \alpha 5(56.9 \mathrm{kDa})$ and Agam $\alpha 5$ $(56.7 \mathrm{kDa}){ }^{33,41,54)}$

The $\mathrm{Md} \alpha 5$ proprotein sequence is most similar to Drosophila D $\alpha 5, \mathrm{D} \alpha 7$, Anopheles Agam $\alpha 5$, Agam $\alpha 7$, and Tribolium $\operatorname{Tc} \alpha 7$. $\operatorname{Md} \alpha 5$ is most closely related to $\mathrm{D} \alpha 5$ because both have an extended $\mathrm{N}$-terminal extracellular domain, and because of the high percentage of $\mathrm{C}$-terminal sequence identity. Although the N-terminal sequence is variable, when the 503-aa C-terminal sequence was compared (pair wise) with the mature protein of other insect nAChRs, it showed $95 \%, 86 \%, 79 \%, 79 \%$ and $78 \%$ identity with D $\alpha 5$, Agam $\alpha 5$, $\operatorname{Agam} \alpha 7, \operatorname{Tc} \alpha 7$ and $\mathrm{D} \alpha 7$, respectively.

Thirteen A-to-I editing sites were identified in $M d \alpha 5$ and 11 sites resulted in amino acid substitution. ${ }^{38)}$ These sites are all situated in transmembrane domains. Two are located in TM3 and nine in the TM4 domain (Fig. 3). The A-to-I RNA editing at nt 1704, 1705, 2278 and 2295 are conserved, both in terms of location and editing rate in $M d \alpha 5$ and $\mathrm{D} \alpha 5,{ }^{45)}$ but these sites are not edited in Agam $\alpha 5$ of An. gambiae or Amel $\alpha 5$ of Apis Melliferai. ${ }^{33,34)}$ There was no difference in Ato-I editing between susceptible and resistant strains that could be associated with resistance. ${ }^{38)}$

Expression of $M d \alpha 5$ in the head, thorax and abdomen of aabys adult flies was investigated using quantitative real-time PCR. Md $\alpha 5$ expression was 340- and 23-fold higher in the head and thorax, respectively, relative to the abdomen. ${ }^{38)}$ Expression levels did not vary between susceptible and spinosad resistant strains.

With the use of polymorphisms in the $M d \alpha 5$ genomic sequences it was shown that this gene is on chromosome 1 of the house fly. ${ }^{38)}$ This result is in agreement with the Drosophila/Musca homology map. ${ }^{53)}$

\section{Characterization of $M d \beta 3$ from Susceptible and Spinosad Resistant House Flies}

The full length (1744 bp) cDNA of $M d \beta 3$ consists of a 1296bp ORF which encodes a 432-aa proprotein that possesses a 26-aa signal peptide. ${ }^{38)}$ Besides the signal peptide, this protein has typical features of nAChRs including a long N-terminal extracellular domain, the Cys loop domain and four hydrophobic transmembrane domains (TM1-4). It lacks the cysteine doublet in the N-terminal extracellular domain and therefore, this subunit is classified as a non- $\alpha$ or $\beta$ subunit. Similar to $\mathrm{D} \beta 3, \mathrm{Md} \beta 3$ has an extremely short C-terminal extracellular domain of only one amino acid. ${ }^{38)}$ The predicted mature $\operatorname{Md} \beta 3$ protein has a calculated molecular weight of $46.7 \mathrm{kDa}$ and an isoelectric point of 5.08 , similar to $\mathrm{D} \beta 3$ $(45.3 \mathrm{kDa}$ and $\mathrm{pI}=5.22) .{ }^{55)}$

$\operatorname{Md} \beta 3$ is most similar to D. melanogaster $\mathrm{D} \beta 3, D$. pseudoobscura D $\beta 3$, T. castaneum Tc $\alpha 10$, An. gambiae Agam $\alpha 9$, and the pufferfish Takifugu rubripes Fr $\alpha 4$, having $54 \%, 54 \%, 34 \%, 31 \%$ and $19 \%$ identity (based on comparison of mature protein sequences), respectively. Analysis of genomic DNA sequences revealed that $M d \beta 3$ contained no introns. ${ }^{38)} M d \beta 3$ is unique in this regard, as all other insect nAChR subunit genes have introns (e.g. $D \beta 3$ has 3$){ }^{33,55)}$

Three alleles were determined by analyzing genomic sequences. Two alleles were found (alleles A and B) in aabys and two alleles (allele B and C) in rspin. There are 27 SNPs between alleles A and B, 2 SNPs between allele B and C and 29 SNPs between allele A and C. Only one (G1231A) of them resulted in an amino acid substitution (V411A). Nucleotide sequence comparison between the aabys and rspin strains did not reveal any specific nucleotide difference associated with spinosad resistance, indicating that differences in the $M d \beta 3$ alleles are not responsible for spinosad resistance. No A-to-I RNA editing was found in $M d \beta 33^{38)}$

$M d \beta 3$ expression was 2.4-fold higher in the head but 1.3fold lower in the thorax, respectively, relative to the abdomen. The expression of these two genes was not significantly different $(P>0.05)$ in aabys and rspin flies. ${ }^{38)}$

\section{Mechanism of Spinosad Resistance in House Flies}

Spinosad resistance in house fly is a recessive trait that could not be overcome with insecticide synergists, suggesting resist- 
ance was caused by an altered target site encoded by a locus on autosome $1{ }^{13)}$ Drosophila $D \alpha 5, D \alpha 6$ and $D \beta 3$ homologs were assumed to reside on autosome 1 according to the Drosophila/Musca homology map. ${ }^{53)}$ Md $\alpha 5$, Md $\alpha 6$ and $M d \beta 3$ have been cloned and analyzed from spinosad susceptible and resistant strains. Contrary to our expectation, the $\mathrm{nAChR}$ subunit genes on autosome 1 were not different between insecticide susceptible and spinosad resistant strains, suggesting that spinosad resistance is not due to a mutation in these nAChR subunits. It is possible that the house fly could have a unique nAChR subunit gene on chromosome 1 , but without a genome sequence this would be challenging to determine. Could spinosad resistance be due to a mutation in a GABA gated chloride channel (i.e. in the secondary target site)? The best studied GABA gated chloride channel in insects is $R d l$ and this gene is on chromosome 4 in house flies, indicating it is not involved in resistance. Given the highly similar characteristics of spinosad resistance in most insects (see above), identification of the mutation responsible for resistance in one species could likely open the door for identification of the mutation responsible for resistance in other species. Unraveling the mystery of spinosad resistance in house flies will require further study.

\section{References}

1) B. L. Bret, L. L. Larson, J. R. Schoonover, T. C. Sparks and G. D. Thompson: Down to Earth 52, 6-13 (1997).

2) G. D. Thompson, R. Dutton and T. C. Sparks: Pest Manag. Sci. 56, 696-702 (2000)

3) A. Paul, L. C. Harrington and J. G. Scott: J. Med. Entomol. 43, 55-60 (2006).

4) J. G. Scott: Pestic. Sci. 54, 131-133 (1998).

5) J. G. Bond, C. F. Marina and T. Williams: Med. Vet. Ent. 18, 50 56 (2004).

6) V. L. Salgado, J. J. Sheets, G. B. Watson and A. L. Schmidt: Pestic. Biochem. Physiol. 60, 103-110 (1998).

7) V. L. Salgado: Pestic. Biochem. Physiol. 60, 91-102 (1998).

8) N. Millar and I. Denholm: Invert. Neurosci. 7, 53-66 (2007).

9) V. L. Salgado and T. C. Sparks: "Comprehensive Molecular Insect Science,” ed. by L. I. Gilbert, K. Iatrou and S. S. Gill, Elsevier, Boston, pp. 137-173, 2005.

10) V. L. Salgado: Down to Earth 52, 35-43 (1997).

11) G. B. Watson: Pestic. Biochem. Physiol. 71, 20-28 (2001).

12) T. Perry, J. A. McKenzie and P. Batterham: Insect Biochem. Molec. Biol. 37, 184-188 (2007).

13) T. Shono and J. G. Scott: Pestic. Biochem. Physiol. 75, 1-7 (2003).

14) M. Kristensen and J. B. Jespersen: J. Econ. Entomol. 97, 10421048 (2004)

15) J. Hsu and H. Feng: J. Econ. Entomol. 99, 931-936 (2006).

16) N. Liu and X. Yue: J. Econ. Entomol. 93, 1269-1275 (2000).

17) Y. Wei, A. G. Appel, W. J. Moar and N. Liu: Pest Manag. Sci. 57, 1055-1059 (2001).

18) H. A. Lester, M. I. Dibas, D. S. Dahan, J. F. Leite and D. A. Dougherty: Trends Neurosci. 127, 329-336 (2004).
19) T. Narahashi: Pharmacol. Toxicol. 78, 1-14 (1996).

20) M. Tomizawa, B. Latli and J. E. Casida: "Nicotinoid Insecticides and the Nicotinic Acetylcholine Receptor," ed. by I. Yamamoto and J. E. Casida, Springer, Tokyo, pp. 271-292, 1999.

21) I. Yamamoto: "Nicotinoid Insecticides and the Nicotinic Acetylcholine Receptor," ed. by I. Yamamoto and J. E. Casida, pp. 327, 1999.

22) S.-J. Lee, M. Tomizawa and J. E. Casida: J. Agric. Food Chem. 57, 2646-2652 (2003).

23) A. Liu, M. S. Williamson, S. J. Lansdell, Z. Han, I. Denholm and N. S. Millar: J. Neurochem. 99, 1273-1281 (2006).

24) Z. Liu, M. S. Williamson, S. J. Lansdell, I. Denholm, Z. Han and N. S. Millar: Proc. Natl. Acad. Sci. USA 102, 8420-8425 (2005)

25) T. Perry, D. G. Heckel, J. A. McKenzie and P. Batterham: Insect Biochem. Mol. Biol. 38, 520-528 (2008).

26) J. A. Dani and D. Bertrand: Ann. Rev. Pharmacol. Toxicol. 47, 699-729 (2007).

27) A. Karlin: Nat. Rev. Neurosci. 3, 102-114 (2002).

28) T. Grutter and J. P. Changeux: Trends Biochem. Sci. 26, 459-463 (2001)

29) D. B. Sattelle, A. K. Jones, B. M. Sattelle, K. Matsuda, R. Reenan and P. C. Biggin: BioEssays 27, 366-376 (2005).

30) A. K. Jones, G. Elgar and D. B. Sattelle: Genomics 82, 441-451 (2003).

31) M. Tomizawa and J. E. Casida: Annu. Rev. Pharmacol. Toxicol. 45, 247-268 (2005)

32) R. F. Chapman: "The Insects Structure and Function," Cambridge Univ. Press, Cambridge, 1998.

33) A. K. Jones, M. Grauso and D. B. Sattelle: Genomics 85, 176187 (2005).

34) A. K. Jones, V. Raymond-Delpech, S. H. Thany, M. Gauthier and D. B. Sattelle: Genome Res. 16, 1422-1430 (2006).

35) A. Jones and D. Sattelle: BMC Genomics 8, 327 (2007).

36) N. S. Millar: Biochem. Soc. Trans. 31, 869-874 (2003).

37) S. P. Foster, N. B. Kift, J. Baverstock, S. Sime, K. Reynolds, J. E. Jones, R. Thompson and G. M. Tatchell: Pest. Manag. Sci. 59, 1169-1178 (2003).

38) J.-R. Gao, J. M. Deacutis and J. G. Scott: Insect Molec. Biol. 16, 691-701 (2007)

39) J.-R. Gao, J. M. Deacutis and J. G. Scott: Arch. Insect Biochem. Physiol. 64, 30-42 (2007).

40) J.-R. Gao, J. M. Deacutis and J. G. Scott: Insect Molec. Biol. 16, 325-334 (2007)

41) M. Grauso, R. A. Reenan, E. Culetto and D. B. Sattelle: Genetics 160, 1519-1533 (2002).

42) Y. Jin, N. Tian, J. Cao, J. Liang, Z. Yang and J. Lv: BMC Evolutionary Biology 7, 98 (2007)

43) A. M. Hosie, S. D. Buckingham, J. K. Presnail and D. B. Sattelle: Neuroscience 102, 709-714 (2001).

44) P. H. Seeburg: Neuron 35, 17-20 (2002).

$45)$ B. Hoopengardner, T. Bhalla, D. Staber and R. Reenan: Science 301, 832-836 (2003).

46) H. Young, W. Bailey and R. Roe: Crop Protection 22, 265-273 (2003).

47) C. Wyss, H. Young, J. Shukla and R. Roe: Crop Protection 22, 307-314 (2003) 
48) J. Z. Zhao, Y. X. Li, H. L. Collins, L. Gusukuma-Minuto, R. F. Mau, G. D. Thompson and A. M. Shelton: J. Econ. Entomol. 95, 430-436 (2002).

49) A. H. Sayyed, D. Omar and D. J. Wright: Pest Manag. Sci. 60, 827-832 (2004).

50) Z. M. Li, S. S. Liu, Y. Q. Liu and G. Y. Ye: Bull. Entomol. Res. 97, 627-635 (2007)

51) J. M. Deacutis, C. A. Leichter, A. C. Gerry, D. A. Rutz, W. D. Watson, C. J. Geden and J. G. Scott: J. Agric. Urban Entomol.
23, 105-110 (2007).

52) N. Liu and J. G. Scott: J. Econ. Entomol. 90, 1478-1481 (1997).

53) G. G. Foster, M. J. Whitten, C. Konovalov, J. T. A. Arnold and G. Maffi: Genet. Res. Camb. 37, 55-69 (1981).

54) P. Wu, D. Ma, M. Pierzchala, J. Wu, L.-C. Yang, X. Mai, X. Chang and T. Schmidt-Glenewinkel: J. Biol. Chem. 280, 2098720994 (2005).

55) S. Lansdell and N. Millar: J. Neurochem. 80, 1009-1018 (2002). 